\title{
The environment ontology in 2016: bridging domains with increased scope, semantic density, and interoperation
}

Pier Luigi Buttigieg ${ }^{1 *}$, Evangelos Pafilis ${ }^{2}$, Suzanna E. Lewis ${ }^{3}$, Mark P. Schildhauer ${ }^{4}$, Ramona L. Walls ${ }^{5}$ and Christopher J. Mungall

\begin{abstract}
Background: The Environment Ontology (ENVO; http://www.environmentontology.org/), first described in 2013, is a resource and research target for the semantically controlled description of environmental entities. The ontology's initial aim was the representation of the biomes, environmental features, and environmental materials pertinent to genomic and microbiome-related investigations. However, the need for environmental semantics is common to a multitude of fields, and ENVO's use has steadily grown since its initial description. We have thus expanded, enhanced, and generalised the ontology to support its increasingly diverse applications.

Methods: We have updated our development suite to promote expressivity, consistency, and speed: we now develop ENVO in the Web Ontology Language (OWL) and employ templating methods to accelerate class creation. We have also taken steps to better align ENVO with the Open Biological and Biomedical Ontologies (OBO) Foundry principles and interoperate with existing $\mathrm{OBO}$ ontologies. Further, we applied text-mining approaches to extract habitat information from the Encyclopedia of Life and automatically create experimental habitat classes within ENVO.

Results: Relative to its state in 2013, ENVO's content, scope, and implementation have been enhanced and much of its existing content revised for improved semantic representation. ENVO now offers representations of habitats, environmental processes, anthropogenic environments, and entities relevant to environmental health initiatives and the global Sustainable Development Agenda for 2030. Several branches of ENVO have been used to incubate and seed new ontologies in previously unrepresented domains such as food and agronomy. The current release version of the ontology, in OWL format, is available at http://purl.obolibrary.org/obo/envo.owl.

Conclusions: ENVO has been shaped into an ontology which bridges multiple domains including biomedicine, natural and anthropogenic ecology, 'omics, and socioeconomic development. Through continued interactions with our users and partners, particularly those performing data archiving and sythesis, we anticipate that ENVO's growth will accelerate in 2017. As always, we invite further contributions and collaboration to advance the semantic representation of the environment, ranging from geographic features and environmental materials, across habitats and ecosystems, to everyday objects in household settings.
\end{abstract}

Keywords: Environmental semantics, Habitat, Ecosystem, Ontology, Anthropogenic environment, Indoor environment, Sustainable development

\footnotetext{
* Correspondence: pier.buttigieg@awi.de

${ }^{1}$ Alfred Wegener Institut, Helmholtz Zentrum für Polar- und

Meeresforschung, Am Handelshafen 12, 27570 Bremerhaven, Germany

Full list of author information is available at the end of the article
} 


\section{Background}

An environment includes the natural or anthropogenic systems which can surround a living or non-living entity. This broad definition encompasses an enormous diversity of entities and scales, thus presenting numerous challenges for constructing ontologies and standards. Previously, we described the Environment Ontology (ENVO; [1]), a community-driven project which represents environmental entities including biomes, environmental features, and environmental materials. At that time, our focus was primarily on representing the environments associated with metagenomic samples: our goal was to provide a vocabulary with which to characterise sequenced environmental samples, together with an ontological structure to facilitate search, advanced querying, and inference in support of the aims of the Genomics Standards Consortium (GSC; [2]). This previous version of the ontology contained a variety of classes for describing a sample along three primary axes: the biome or ecosystem within which an entity of interest (usually an organism or community) is embedded; the environmental features that are in the vicinity of and have a strong causal influence on the entity; and the environmental material that is the substance surrounding or partially surrounding the entity. Although the use case is primarily microbial, the approach can encompass larger organisms - for example, a killer whale in a neritic epipelagic zone biome, present in an ecosystem defined by a marine subtidal rocky reef, and surrounded by coastal water. We also described the dynamic nature of the ontology, and the process for community extension of the ontology.

\section{New challenges}

In the time since our initial publication, we have oriented ENVO's development to a suite of emerging challenges extending our original and core case of describing samples of environmental and biomedical importance (e.g. [3-5]). On the one hand, sequencing projects are targeting ever more diverse environments such as city transit systems [6] and also phenomena such as soil compaction in forest ecosystems [7]. This has driven new requests from adopters such as MG-RAST [8] and the iMicrobe project (http://imicrobe.us/) which has annotated some 2813 environmental metagenomic samples with ENVO terms (see http://data.imicrobe.us/ and [9]). On the other hand, we have encountered a number of entirely new use cases in areas such as ecology and biodiversity science. Both of these fronts have, at times, required the expansion of existing branches in the ontology and, at others, required the creation of either entirely new branches, or the refactoring of existing branches. This increase in scope also presented challenges and opportunities in terms of how the ontology should be interwoven with other ontologies in the OBO Foundry and Library (http://obofoundry.org/) [10].

In this update, we describe how we have extended and in some cases broken apart ENVO to meet the above challenges. We also describe how these efforts have connected ENVO to a broader movement to further extend OBO-aligned semantics into the realm of ecology and biodiversity science [11-13], centred on co-development with ecologically themed ontologies such as the Population and Community Ontology (PCO) and Bio-collections Ontology (BCO) [14]. These efforts have been catalysed by several workshops and meetings e.g. [4] which have greatly supported ENVO in contending with entities such as habitats, environmental processes, and environmental dispositions while orienting its content to address issues of global importance.

\section{Expanding usage and coordination}

Along with its scope, the use of ENVO is also growing and supporting data annotation, searching of datasets, and the mobilisation of sample data. For example, the journal Scientific Data (Nature Publishing Group; ISSN 2052-4463) now uses ENVO classes to annotate its Data Descriptor articles [15], allowing articles to be browsed with faceted interfaces (http://scientificdata.isa-explorer.org), and PANGAEA, a data publisher for Earth and environmental science, is continuing to use the ontology to enrich its metadata and data archives (http://www.pangaea.de). Parallel efforts such as those convened by the Global Biodiversity Information Facility (GBIF) have moved to enhance the widely used Darwin Core (DwC; http:// rs.tdwg.org/dwc/; [16]) glossary by using ENVO in habitat descriptions [17]. Other users have begun to explore ENVO's potential in data analysis [18] and in contributing to semantically aware biodiversity informatics (e.g. [19, 20]). Further, synthesis centres such as the National Centre for Ecological Analysis and Synthesis (NCEAS; Santa Barbara, USA; http://nceas.ucsb.edu/) and the Centre de synthèse et d'analyse sur la biodiversité (CESAB; Aix-en-Provence, France; http://cesab.org/) have engaged with us to explore further possibilities for usage and provide advice on coordination and community needs linked to projects such as the Data Observation Network for Earth (DataONE; www.dataone.org). Indeed, it is the diverse needs of these communities, as well as those of more recent partners (see Results and Discussion), which have compelled ENVO to develop with generalisability and versatility in mind, as is appropriate for a domain or reference ontology. Representations of microscale environments co-exist and interoperate with those of planetary-scale systems and are being further harmonised as the ontology grows in scope. 


\section{An overview of this update}

Below, we describe the updates made to improve ENVO's ability to maintain coherence while meeting the needs of its diversifying user base and implementation partners. Our Methods section describes key technical updates while our Results focus on content-level changes. The first section of our results describes ENVO's increased expressivity, acquired through transitioning to a more powerful development language. The second section describes the addition of processes to ENVO's content, which has widened ENVO's range of application and enriched the relationships between its classes. Building on its updated expressivity, the third section describes how ENVO distinguishes between environments and habitats and how thousands of habitats linked to species descriptions have been represented using text-mining approaches. Departing from the natural setting, the fourth and fifth sections describes the increased efforts made in representing anthropogenic or anthropised environments and how these changes relate to the monitoring of policy objectives and global development. Finally, we comment on how ENVO intends to handle its rapidly growing scope while maintaining expert-guided representations. From a wider perspective, we believe these updates represent multi-stakeholder convergence on the goal of integrating data through environmental contextualisation across the biosphere.

\section{Technical note}

As a technical note, the reader is advised that OBO Library ontologies are assigned unique acronyms or initialisations, such as BFO or ENVO, that serve as shorthand identifiers for that ontology. In the following text, ontology classes (or, synonymously, 'terms'), are written in italics and are taken from ENVO unless otherwise marked through the provision of an appropriate ontology prefix, as in 'PATO:laminar'. The unique shorthand fragment of each term's Permanent Uniform Resource Locator (PURL), e.g. 'ENVO_00002297' for 'environmental feature', will be included on first mention of any class, in which case the redundant namespace prefix shall be omitted. Full PURLs are of the form: http://purl.obolibrary.org/obo/ ENVO_00002297, and are resolved to OWL as well as to human-readable web pages via OntoBee [21].

\section{Methods}

The development of ENVO is now conducted using Protégé (http://protege.stanford.edu), rather than OBO Edit [22], allowing more expressivity through the Web Ontology Language (OWL). For global interoperability, we preferentially use relations from the Relations Ontology (RO; [23]) and the Basic Formal Ontology (BFO; [24]) to connect these classes. Additional relations are present, but will be incorporated into RO pending an open discussion and vetting process. The ontology is still released in both OBO and OWL formats and a number of custom exports have been made upon request (e.g. flat, character delimited formats suitable for import into relational databases, tableoriented analysis software, or network visualisation and analysis solutions). We continue to maintain obsoleted terms and link them to their replacements (where available) in a machine readable way to support automated updating of user implementations.

As with most other OBO Library ontologies, ENVO's repository has been moved to its own GitHub "organization" (https://github.com/EnvironmentOntology). This change does not affect downstream users who consume the ontology using standard permanent URLs; however, it does provide a better mechanism for stakeholders to become involved with the development of the ontology through, for example, an improved issue tracker [25]. Further, it allows easier reference to previous versions of the ontology for backwards compatibility.

OWLTools (https://github.com/owlcollab/owltools) and ROBOT [26] (https://github.com/ontodev/robot/) are currently being used for release management, and for the import of classes from other OBO Foundry and Library ontologies in alignment with the Minimum Information to Reference an External Ontology Term (MIREOT; [27]) guidelines. These import procedures are primarily used to express environments that are dependent on entities defined outside of ENVO. For example, environments defined by anatomical entities and chemical entities are expressed using classes from ontologies such as the Uber Anatomy Ontology (UBERON; [28]) and the Chemical Entities of Biological Interest Ontology (CHEBI; [29]) to prevent duplicating existing, well-developed semantics relevant to terms such as 'xylene contaminated soil' [ENVO_00002146] and 'axilla skin environment' [ENVO_08000001].

We have created a TermGenie instance (http://envo. termgenie.org/) [30] that allows for web-based addition of new terms that conform to a pre-defined template, or following a free-form pattern. We are also documenting our design patterns (ODPs) using the emerging 'dead simple owl design patterns' standard (https://github.com/ dosumis/dead_simple_owl_design_patterns) and are using these patterns to generate small portions of the ontology. Further, we have begun to use the results of text-mining approaches, noted in [1], discussed below, and documented by Pafilis et al. [31], to automatically generate experimental classes which, upon curation, can be integrated into the core ontology.

\section{Results and discussion}

ENVO now includes some 2159 classes primarily representing biomes, geographic features, and environmental materials, along with 18,791 axioms (logical statements) 
defining, interconnecting, and interrelating them. This contrasts with 1644 classes and 14,542 axioms present when ENVO's original description was published. The growth of the ontology was primarily driven by the needs of the 'omics community using the Minimal Information about any (x) Sequence (MIxS; [32]) checklist and its extensions such as MIxS for the Built Environment (MIxS-BE; [33]). These needs were communicated through individual requests for new classes and requests coordinated through, for example, curation efforts of organisations such as the European Nucleotide Archive (ENA) (e.g. [34]). More currently, the bulk of the changes to ENVO's content have been motivated by the ontology's growing adoption and engagement with new user communities as well as the need to integrate their varying approaches to describing environments.

\section{Increases in semantic density and expressivity}

As we are now developing ENVO using the expressivity of OWL (see Methods), we have increased the variety and density of linkages between many of ENVO's classes as well as the detail in their logical definitions. This increased semantic density offers more flexibility when using the ontology for querying, inference, and semantically enhanced analysis. To illustrate the increased expressivity, an oasis [ENVO_01001304] (Fig. 1) is represented as a subclass of 'vegetated area' [ENVO_01001305] which has, as a part, some 'spring' [ENVO_00000027] and is partially surrounded by a portion of either rock [ENVO_00001995], sand [ENVO_01000017], or soil [ENVO_00001998] which, itself, is arid [ENVO_01000230]. This representation has several facets which involve type hierarchy (i.e. class and subclass relationships), parthood, and adjacency, and which define key properties of one or more of the classes involved. Practically, users and machine agents can now identify an oasis (and any data that has been associated with that class) by any one of these routes such as querying for a vegetated area that is surrounded by arid environmental materials or which has a spring as a necessary part.

The increased axiomatisation described above has also improved our ability to represent semantically problematic classes such as 'hydrographic feature' [ENVO_00000012] and 'marine pelagic feature' [ENVO_01000044]. The issues with these somewhat artificial or convenience groupings are discussed in [1]; in brief, their membership is dictated more through convention than physical or formative similarities, often adding ambiguity and confounding search and inference. For example, one is correct in asserting that a lighthouse, a lake, and a coral reef are hydrographic features due to the nautical conventions of hydrography; however, these entities are substantially different from one another and much better distributed in hierarchies true to their physical attributes and/or the processes of their formation. With ENVO's greater semantic flexibility, the varied criteria for including a class in one of these convenience groupings can be more precisely defined and classes which satisfy these criteria can be interlinked through automated inference: the action of reasoning software which can use logical statements to infer relationships and hierarchies which were not asserted by a human. For example, any class which has been asserted to be 'adjacent to' some 'water body' or 'partially surrounded by' some 'water' will be inferred to be a subclass of 'hydrographic feature'. Similarly, 'marine pelagic feature' would be populated by any entity which has been asserted to be 'part of' some 'marine water body' or 'composed primarily of' some 'sea water'. Similarly, many subclasses of 'environmental material' [ENVO_00010483] are now placed in inferred hierarchies using various subclasses of quality [PATO_0000001] such as 'quality of a solid' [PATO_0001546], 'quality of a gas' [PATO_0001547], and 'quality of a liquid' [PATO_0001548]. Such assertions provide a way to construct and populate classes like

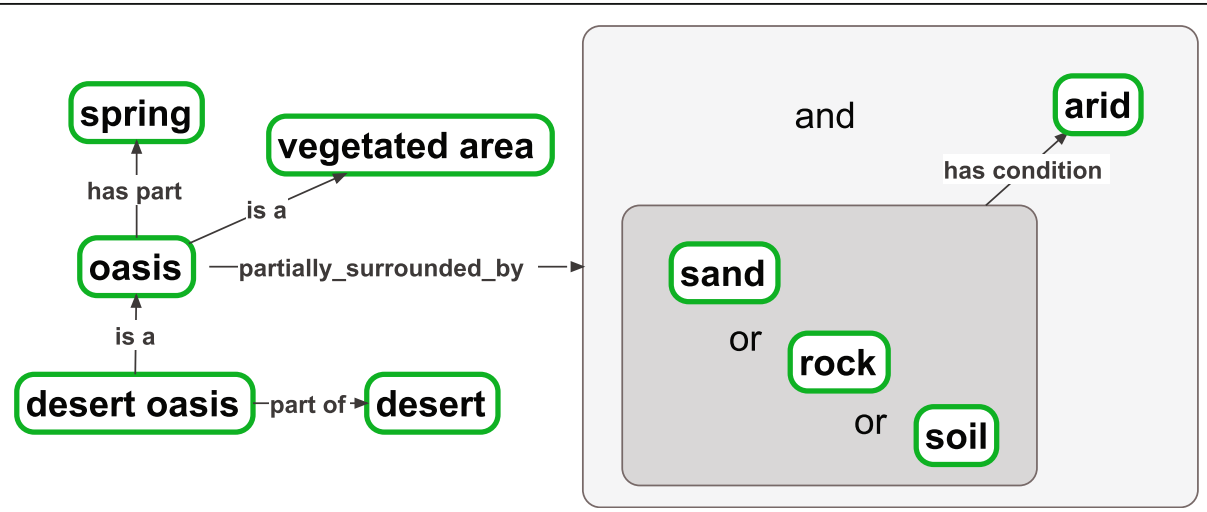

Fig. 1 Illustrative example of ENVO's improved semantic expression with OWL axioms. An oasis is a vegetated area which has, as a part, a spring and is surrounded by an arid portion of soil, rock, or sand 
"solid" or "liquid" through inference, avoiding asserted multiple inheritance while simultaneously preserving clear representations based on multiple criteria.

As illustrated above, the flexibility that comes with increased axiomatisation is an important step in supporting multiple, varying classifications of environmental entities in an integrated fashion. We will leverage these capacities to disentangle the semantics of environmental entities across user groups which use different definitions for syntactically similar terms. The hundreds of official and operational definitions of "forest" [35], which can influence critical decisions in conservation and sustainable land use [36, 37], will be one of our first targets in this process. We anticipate that ENVO will host multiple classes representing the different entities typically gathered under one label, using synonym lists and crossreferences to relevant definition sources to untangle alternative term usage. This approach will allow a diversity of users, including those with limited exposure to semantic technology, to easily identify which class they wish to employ. Simultaneously, advanced users can take advantage of ENVO's continually developing axiomatisation to perform analyses of such semantic spaces and to mobilise data in novel ways. In addition to axiomatisation of relatively static entities (or continuants [BFO_0000002]), we aim to further extend this flexibility through the representation of processes, described in the following section.

\section{Representing environmental processes}

The material parts of environments are constantly changing and the representation of the processes which are involved in such changes naturally falls in ENVO's scope. Consequently, an initial set of some 53 classes representing environmental processes, aligned with the process [BFO_0000015] class in the Basic Formal Ontology, have been added to ENVO and have been used to interlink material entities throughout the ontology. As an example, a 'volcanic eruption' [ENVO_01000634] ties together magma [ENVO_01000648], lava [ENVO:010 00231], tephra [ENVO:01000660], and a set of gaseous materials through relations of input, output, and more general participation (Fig. 2). Inference (described above) can be used to populate processes such as 'carbon-bearing gas emission process' [ENVO_01000742] with both natural and anthropogenic processes based on their inputs and outputs. Such constructions can be used to efficiently represent higher-order processes such as 'climate change' [ENVO_01000629]. The relations between these classes are primarily controlled by the Relations Ontology (RO, [23]) and work is underway to update both ENVO and RO to offer more powerful expression.

Further, processes can be used to define entities which arise as a result of their instantiation in a more machineactionable manner. For example, an igneous intrusion process' [ENVO_01000657] may be linked to an 'igneous intrusion' [ENVO_01000659] through RO's 'formed as a result of" [RO_0002354] relation. Historically, many of the $\mathrm{RO}$ relations connecting processes with independent continuants have primarily been applied to the biological process hierarchy of the Gene Ontology [38], and may require some generalisation for environmental processes. We hope to expose these as ENVO's process hierarchy develops and lead the extension of relational semantics into ecological and environmental domains.

In addition to their immediate utility, classes representing environmental processes allow a key point of interaction with other ontologies and semantic resources. To illustrate, participation in a 'land consumption process' [ENVO_ 01000743] may encompass material and immaterial anthropogenic and natural entities such as: buildings; legal documents and rights; indigenous populations and lands; and ecosystems. This will be essential to interweave ontologies across broad "super-domains" such as sustainable development (see Environmental Semantics in support of the Sustainable Development Agenda for 2030, below) as well as articulate threats to habitats.

\section{Clarifying and representing habitats}

Interest in a given environmental system and the processes which change it is often driven by the desire to understand the ecology of the organisms that inhabit it. The relationship between populations of organisms and the one or more environmental systems needed to sustain their existence and growth is the foundation for the semantics of "habitat". ENVO's previous representation of habitats was underdeveloped, and many of its classes confounded the semantics of "environment" and "habitat", primarily due to the loose usage of these terms across disciplines. Thus, as anticipated by Buttigieg et al. [1], ENVO's semantically confounded habitat [ENVO_00002036] class has been made obsolete and replaced by the equivalently labelled, habitat [ENVO_01000739]. ENVO's current habitat class represents an environmental system within which an ecological population (i.e. population [PCO_0000001]), can persist and grow. Importantly, a population of a given species (or similar grouping) need not be present in such an environmental system in order for that system to qualify as that species' habitat: the environmental system need only have the disposition to support such a population.

Typically, subclasses of the current habitat will be formulated similarly to 'Equus zebra habitat', in that they will always reference some species or other grouping of organisms with similar physiological tolerances and environmental preferences. Habitats can be related to their constituent environments using the overlaps [RO_0002131] relation, as any given habitat will share parts with a range of environment types, according to the requirements of the species of interest. Organisms and populations of 


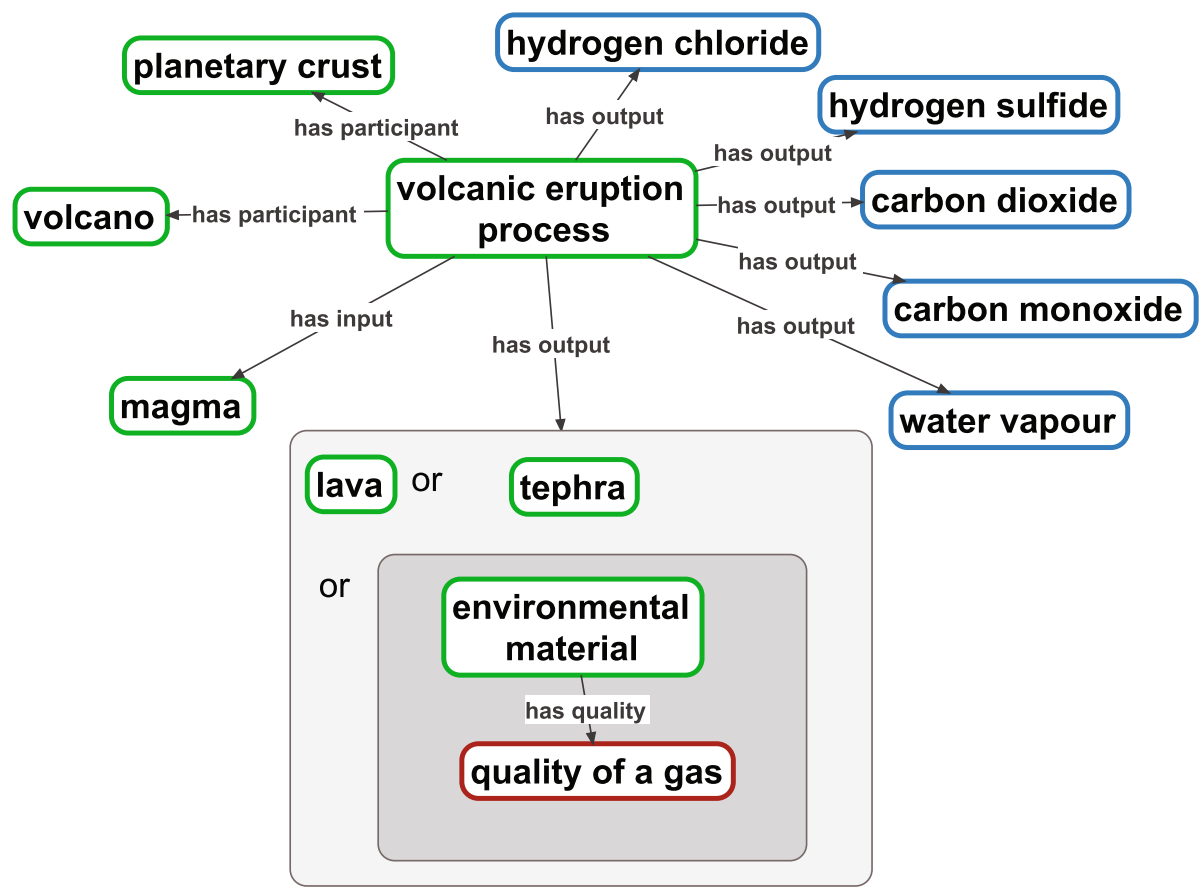

Fig. 2 Illustrative example of ENVO's representation of a volcanic eruption process. ENVO's new environmental process branch allows the participants, inputs, and outputs of environmental processes to be represented. These entities may be from ENVO's content or imported from other ontologies, promoting interoperation and orthogonality. Green borders indicate ENVO classes, blue borders CHEBI classes, and red borders PATO classes

organisms can be associated with their habitats by the 'has habitat' [RO_0002303] relation, the definition of which was updated as a result of ENVO's clarified representation of habitat. See Fig. 3 for illustration of these semantics.

As most collections of organisms grouped at species (and even sub-species) level can be associated with a distinct habitat, the number of classes in this branch is likely to become very large and automated approaches are required to make reasonable progress. Thus, we created an experimental branch of ENVO based on the results of the ENVIRONMENTS-EOL project [31], which text-mined the habitat descriptions of the Encyclopedia of Life [39] and associated them with ENVO classes. This approach generated results for 227,583 taxa, associating them with 1,605,974 automatically generated annotations ("tags") based on ENVO class labels and synonyms. We reduced this collection to 112,585 taxa by removing taxa which we were unable to link to a National Center for Biotechnology Information (NCBI) taxonomy entry via the EOL API. This filtering was performed to focus on taxa that we could readily map to a widely-used taxonomy which is integrated with genomic data. We acknowledge that other taxonomies and/or phylogenies may be more accurate, both globally and for specific taxa: initiatives such as the Tree of Life Web Project [40], PhylomeDB [41], and TreeBase [42, 43] are of great interest in enhancing this dimension of our habitat hierarchy, and we will work towards integrating additional taxonomic resources in future releases. We then chose to focus our attention on taxa which face threats to their persistence by retaining only those taxa which feature in the International Union for Conservation of Nature (IUCN) Red List [44] as extinct in the wild (EW), regionally extinct (RE), critically endangered (CR), or endangered (EN), yielding 5,117 taxa. Due to their experimental nature, the results of this exercise are stored in a separate repository [45] and classes exist in a temporary ID range (prefixed with "ENVO:H"). The complete result set may be retrieved from [46].

Our automatic mapping provides a foundation upon which high-quality semantic resources can be created for linking organisms to the environments which sustain their populations. However, this automatic mapping is prone to error and must be refined. Erroneous mappings have been identified due to simple false positives, ambiguous class labels, and text-mining routines which only account for the basic structure of the ontology. False positives can easily arise from the parsing of place names such as "Mountain River" or from other largely unpredictable facets of natural language. An example of the latter two issues was apparent in the overly narrow association of class label 'pelagic zone' [ENVO_00000208] to marine ecosystems. Large lakes are also said to have pelagic zones, however, workers in both marine and lacustrine domains will generally omit labels with qualifiers such as "marine pelagic zone". Within ENVO, we decided to err 


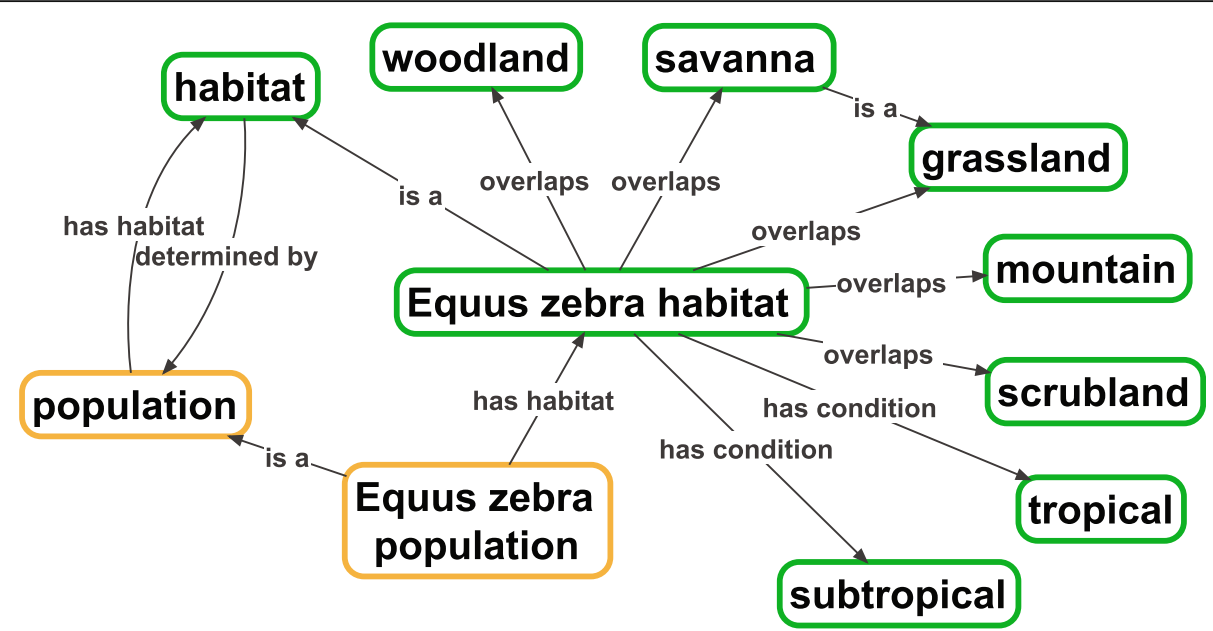

Fig. 3 Typical formulation of an experimental habitat class in the ENVO's automatically generated habitat content. Here, the environmental system which can support the survival and growth of a population of Equus zebra is represented. Based on the Encyclopedia of Life's descriptions of the environments in which such populations are typically found, their habitat is represented as an environmental system which overlaps these environments. The boundaries of the habitat are determined by the physiological tolerances of the organisms: the habitat ends where the potential for an organism (or mating pair of organisms) to survive and/or increase their population size is no more. See text for comments on handling different definitions of 'habitat'. Green borders indicate ENVO classes, while orange borders represent PCO classes

on the side of caution and employ such modifiers, maintaining "pelagic zone" as a broad synonym associated with each class. Enhanced text-mining techniques, such as natural language processing (NLP), statistical analysis of text-mining results, and additional filtering based on a term's ontological context, could further reduce false positives. We have yet to explore the feasibility of this solution with a rapidly developing ontology like ENVO, but we are encouraged by the promise of semi-automated ontology growth in the environmental domain.

While ENVO's preliminary habitat representation shows promise, we stress that refinement and curation are needed before habitat classes will be added to the release version of the ontology. We will solicit input from experts on particular species and their environmental preferences in order to validate our mapping, report poor representations, and request enhancements via the ENVO issue tracker [25]. Building on these initial results, we aim to enable semantically controlled, large-scale habitat analyses driven by text-mining as described by authors such as Groom [47]. Eventually, we anticipate that coupling habitat semantics with distributional e.g. [48], trait e.g. [19], or behavioural data will offer further opportunities in predicting multi-scale patterns of biodiversity.

Importantly, it must be acknowledged that there will be some ambiguity in what constitutes an ecological population and, hence, what environments can provide a habitat for its members. Further, definitions of "habitat" also vary (see, e.g. $[49,50]$ ), increasing the need for structured representation of the semantics behind the entity. These issues are further complicated by the decoupling of phylogeny from function due to, for example, horizontal gene transfer in microbes as well as procedural issues in stably identifying units of diversity [51-53] along with the role of microdiversity [54, 55]. Definitional variation and ambiguous boundary conditions are not unusual in the representation of environmental entities. ENVO will remain agnostic regarding any definition's 'correctness' and we anticipate that coexisting and semantically overlapping habitat classes will emerge to represent the entities referenced by different communities. Addressing this challenge will be greatly helped by ENVO's increased semantic flexibility, described in the sections above, which will be leveraged to tease apart this space. Through this process of representation, we hope that ENVO will serve as a hub for healthy and structured debate over central ecological entities such as habitats and niches, while simultaneously providing a resource to mobilise data in transparent ways. As a final but important note, we frequently encountered information indicating the typically deleterious impact of human activity on habitats. This, along with the need to provide semantics for defining anthropised environments, has motivated updates in ENVO's representation of human-centric environmental systems and processes, as we describe below.

\section{Anthropogenic environments and impacts}

Much of ENVO's recent development has been guided by requests for the representation of anthropogenic environments. This is bolstered by the clear need to provide semantics for the interplay of human activity with 
natural systems, echoing Ellis and Ramankutty's call for ecologists to increase their focus on anthropised environments which now dominate the Anthropocene Earth [56]. To illustrate, requests linked to the Program for Resistance, Immunology, Surveillance and Modeling of Malaria in Uganda (PRISM), a project in the framework of the International Centers of Excellence for Malaria Research (ICEMRs; see e.g. [57] for context), have motivated the creation of classes representing housing materials, building components, and building types relevant to the assessment of malaria risk [58]. Examples include concrete [ENVO_01000458], 'sheet-iron building roof' [ENVO_01000510], and 'ventilated improved pit latrine' [ENVO_01000530]. With similar motivation, classes of vehicle [ENVO_01000604] and classes for mesoscopic objects such as lamp [ENVO_01000566] and lantern [ENVO_01000565] have also been added.

Additions motivated by PRISM demonstrate how ENVO's content has been shaped by the needs of an environmental health initiative. We plan to link such efforts to the representation of pathogen or vector habitats to draw together knowledge on the build environment and pathogen ecology. Methods such as DISEASES [59] and Bio-Lark [60] are particularly promising in this regard, leveraging text-mining to discover and link terms from the medical and biological domains. ENVO classes such as slum [ENVO_01000653] and factory [ENVO_01000536] can complement these use cases and reinforce the representations of anthropogenic environments. This work will also produce content which addresses the needs of projects investigating the microbiomes of indoor environments [61-65]. Classes representing building parts such as 'living room' [ENVO_01000423], patio [ENVO_01000424], and 'indoor kitchen' [ENVO_ 01000421] are being used in the annotation of metagenomes [34] and exemplify a convergence of needs which provide a foundation for broad interoperability through environmental semantics.

In parallel to object-type classes, ENVO's material hierarchy is being populated with anthropogenic materials. The ontology has been identified as a means to support the assessment of nanomaterial risk in environmental systems [66] and has classes which are immediately useful. For example, classes such as carbon nanotube enriched soil' [ENVO_01000427] combine soil [ENVO_00001998] and 'carbon nanotube' [CHEBI_50594] in a pattern which is easily propagated. Materials associated with health concerns, such as 'fine respirable suspended particulate matter' [ENVO_01000415] (i.e. PM 2.5), have been added and will be integral to ENVO's role in environmental monitoring efforts (see below). Our aim is to provide semantics capable of supporting the clarification and interoperability of information used to assess human impacts on ecosystems e.g. [67], while promoting collaboration between environmental and material researchers and refining ENVO's content.

Taken with ENVO's increased coverage of natural environments, these updates have prepared the ontology to address challenges in planetary monitoring across scales. We have begun to realise this potential through engagement with the Sustainable Development Agenda for 2030, summarised below.

\section{Environmental semantics in support of the Sustainable Development Agenda for 2030}

Over the course of 2015 and in collaboration with the United Nations Environment Programme (UNEP), the Sustainable Development Goal Interface Ontology (SDGIO; [68]) has been founded with the aim to provide a semantic resource for the Sustainable Development Goals (SDGs, [69-71]), their targets, and indicators. Environmental semantics strongly feature in this effort and ENVO - a key component of SDGIO - is being shaped by its demands. ENVO's increased axiomatisation, process semantics, and representation of anthropogenic environments (described above) will be brought to bear to represent the entities associated with terms across multiple SDGlinked official vocabularies such as the General Multilingual Environmental Thesaurus (GEMET; [72]). To illustrate, we have created processual classes expressing environmental hazards and disasters such as earthquake [ENVO_01000677], tsunami [ENVO_01000689], 'volcanic eruption' [ENVO_01000634], and drought [ENVO_1000745] as well as continuant classes such as 'atmospheric water vapour' [ENVO_01000268], linked to roles such as 'greenhouse gas' [CHEBI_76413], to support the handling of information for Target 13.1 ("Strengthen resilience and adaptive capacity to climaterelated hazards and natural disasters in all countries") of SDG 13 ("Take urgent action to combat climate change and its impacts"). These new categories will be supported by leveraging ENVO's representation of environmental conditions to address the semantics of weather and climate. Further, we have introduced classes representing forest types (relevant to Targets in SDG 15, Targets 6.6), which are being aligned to definitions in the Global Forest Map (GFM) 2000 [73]. Anthropogenic and anthropised environmental entities as well as axioms tying together continuants and processes, discussed above, will play an especially important role in addressing many of the SDGs. We will continue to add content to support the SDGs in all of ENVO's branches and invite the wider community to participate via our issue tracker [25].

The enhancements above have set the foundation for interlinking data described with ENVO to global policy targets through SDGIO via constructs such as ecosystem functions and services. We aim to develop this capacity and facilitate the exposure of scientific outputs to the 
policy community as they become increasingly driven by data products. Early work exploring this potential is underway and aims to semantically annotate and expose outputs of the Frontiers in Arctic Marine Monitoring (FRAM; http://fram-data.awi.de/) programme [74] with ENVO and SDGIO, linking data about fragile Arctic ecosystems to SDGs 13 and 14 ("Conserve and sustainably use the oceans, seas and marine resources for sustainable development"). We encourage other projects, be they single investigations or observatory-scale endeavours, to contact us should they wish to coordinate similar efforts.

\section{Handling an ever-growing scope}

"The environment is everything which isn't me" Albert Einstein.

It is readily apparent that the range of entities represented in ENVO is expanding very rapidly, well beyond its original objectives within the context of the GSC. In many cases, this expansion is due to a lack of similar resources in domains such as architecture, development, or food and agriculture. As environmental systems can feature an immense range of components, it is valid to extend ENVO's content to address these domains. However, it far more desirable that such entities are represented in independent, expert-led ontologies restricted to a disciplinary domain in order to preserve semantic orthogonality and improve accuracy [75]. This would by no means diminish ENVO's capacities or content: ENVO can readily import classes from such domain ontologies to represent the environments they are components of and preserve its current scope in a more sustainable way. Indeed, this strategy has been successfully applied to ontologies such as the metazoan anatomy ontology, UBERON, which federates with separate ontologies dedicated to non-chordate clades, such as sponges, ctenophores and cephalopods (see Methods).

Driven by the rationale described above, we have recently begun to use ENVO's content to seed new domain ontologies. For example, we are contributing to the launch of a food ontology (FOODON; [76]), to which we have transferred ENVO's 'food product' [ENVO_00002002] classes including amasake [ENVO_00003872], 'bambara groundnut product' [ENVO_0010109], and 'zebra milk' [ENVO_02000018]. Further, we are co-developing agronomy and agriculture related semantics with the newly launched Agronomy Ontology (AgrO; [77]), led by members of the CGIAR (http://www.cgiar.org/) and Bioversity International (http://www.bioversityinternational.org/). As noted above, we are also in the process of launching an application ontology for polar oceanographic, biogeochemical, and biological observation linked to the FRAM programme by enhancing ENVO's content for polar investigations [74]. ENVO's role in the growth of the SDGIO will very likely produce more targets for this approach, such as urban infrastructure systems and disaster response systems. In summary, ENVO is likely to handle its ever-growing and highly diverse content by serving as an incubator for the ontologies of orphan domains, aligning them with the best practices of the OBO Foundry and promoting their interoperation with existing resources. We welcome adopters of these protoontologies and offer assistance in launching new ontologies to sustainably extend an ever more comprehensive semantic layer.

\section{Conclusion and outlook}

The growing interest in and use of ENVO has motivated notable expansion and enhancement of the ontology, while simultaneously creating new challenges. The addition of environmental processes and dispositions has extended ENVO's semantic range and supported our efforts to increase its axiomatic density. We have made progress in representing thousands of habitats using methods driven by text-mining and look forward to refining this content to catalyse efforts to synthesise ecological data with clear semantic representation. Furthermore, we have begun to align ENVO with key themes in global conservation and development. Future efforts will concentrate on the representation of entities described by initiatives such as the IUCN Red List of Ecosystems [78] and relevant to the Sustainable Development Agenda for 2030, demanding a cohesive treatment of environments with varying degrees of human impact. We see these efforts as a contribution towards e-infrastructures able to address the grand challenges of sustainably managing Earth's ecosystems, as articulated by Hardisty et al. [79] and in line with rationale of the Bouchout Declaration's aims of creating open standards for integration and sharing of (http://www.bouchoutdeclaration.org/declaration/) along with the recently released FAIR principles [80].

We anticipate that the path ahead will require greater technical enhancements, contributions from the communities it supports, and a broader team of developers in order to facilitate and expedite its development alongside that of the nascent ontologies nested within ENVO's hierarchies. We have begun to employ tools such as TermGenie [30] and ROBOT [26] to address these needs. Further semantic diversity, leveraging Basic Formal Ontology 2.0 (BFO) classes such as history [BFO_0000182], will be explored to formulate classes representing ecological succession and paleoenvironmental entities. Additionally, we plan greater interaction with initiatives such as GloBI [20] to improve the representation of organismal interactions in environmental systems and to make ENVO's semantics more relevant to archetypal 
ecological data sets. Furthermore, we hope to expand our collaboration with synthesis centres and data integrators and are exploring new possibilities with, for example, the Integrated Digitized Biocollections (iDigBio) National Resource for Advancing Digitization of Biodiversity Collections (ADBC) [81].

As always, we extend an invitation to communities of ontologists, informaticians, domain experts, and other current or new users of the semantic layer to interact with and shape ENVO to their needs. We especially welcome groups wishing to adopt the nascent domain ontologies forming within ENVO and users who are able to test whether ENVO's logical structure can enhance their data analyses. We look forward to broadening and deepening the semantic layer for the environmental sciences.

\section{Downloads}

ENVO's latest release version is available for download [82]. A file including only ENVO classes (envo-basic.obo) is available as well as files with additional classes from ontologies used to construct logical definitions in ENVO (envo.obo and envo.owl). The ontology is available both in OBO and OWL format; however, the OWL format features greater expressivity and is a W3C recommendation for semantic representation of objects on the Web (www.w3.org/2004/OWL/). The OBO Library page for ENVO (http://obofoundry.org/ontology/envo.html) also contains an index of available downloads plus links to various browsers offering ENVO. As before, this ontology is free and open to all users and is distributed under a CC-BY license.

\footnotetext{
Acknowledgements

Foremost, we would like to acknowledge the community of contributors who have driven forward ENVO's development. In particular, we thank the National Center for Ecological Analysis and Synthesis (NCEAS/UCSB) and the NSF SONet Award \#0753144 along with the Phenotype Ontology Research Coordination Network (NSF Award \#0956049) for hosting several meetings key to ENVO's advancement; and John Wieczorek and Robert Guralnick for their input. Additionally, we thank Visotheary Ung for discussions and initial work on representing biogeographic areas and Jie Zheng for requests and comments on PRISM-related classes. We are grateful to UNEP for funding several meetings to link ENVO with the SDGs and, in particular, Jacqueline McGlade, Ludgarde Coppens, and Priyanka DeSouza for their contributions. We are also grateful to the CGIAR and Bioversity International, in particular Medha Devare, Elizabeth Arnaud, Marie-Angelique Laporte, and Céline Audebert for work and meetings associated with the Agronomy Ontology. We also thank Alison Specht and Eric Garnier and CESAB for coordinating efforts to link ecological semantics and biodiversity thesauri. We thank two anonymous reviewers for their input and insight. PLB's work on this project was partially supported through the MicroB3 project, funded by the European Union's Seventh Framework Programme (Joint Call OCEAN.2011-2: marine microbial diversity - new insights into marine ecosystems functioning and its biotechnological potential) under the grant agreement no 287589 and the European Research Council Advanced Investigator grant ABYSS 294757 to Antje Boetius. SL and CJM are supported by the Director, Office of Science, Office of Basic Energy Sciences, of the US Department of Energy (DE-AC0205CH11231). RLW was supported by grant \#GBMF4491 from the Gordon and Betty Moore Foundation and by CyVerse (formerly the iPlant Collaborative) under Award Numbers DBI-0735191 and DBI-1265383. EP was supported by the
}

LifeWatchGreece Research Infrastructure [384676-94/GSRT/NSRF(C\&E)]. Part of this work was conducted using the Protégé resource, which is supported by grant GM10331601 from the National Institute of General Medical Sciences of the United States National Institutes of Health. This work was also conducted using tools and infrastructure developed as part of the Gene Ontology Consortium (U41-HG002223) and the Monarch Initiative (R24-OD011883).

\section{Authors' contributions}

All authors read and reviewed the manuscript. PLB led this development stage of ENVO, wrote the manuscript, and contributed to the development of SDGIO and the nascent Food Ontology and Agronomy Ontology. EP provided input on the ENVIRONMENTS-EOL data set, the retrieval of the related NCBI Taxonomy entries and the IUCN-text derived annotations. RLW contributed to the development of the environmental process branch and the SDGIO. SL provided opportunities and resources, through the Phenotype RCN, that enabled this work: Supporting and facilitating faceto-face meetings and collaborations to develop use cases for the semantics of habitat and environmental processes. CJM provided high level design on the processes branch and worked with EP on the creation of the habitat ontology; along with PLB he oversees the releases of ENVO. All authors read and approved the final manuscript.

\section{Competing interests}

The authors declare that they have no competing interests.

\section{Author details}

${ }^{1}$ Alfred Wegener Institut, Helmholtz Zentrum für Polar- und Meeresforschung, Am Handelshafen 12, 27570 Bremerhaven, Germany. ${ }^{2}$ Institute of Marine Biology Biotechnology and Aquaculture, Hellenic Centre for Marine Research, P.O Box 2214, Heraklion 71003, Crete, Greece.

${ }^{3}$ Environmental Genomics and Systems Biology Division, Lawrence Berkeley National Laboratory, Berkeley, CA 94720, USA. ${ }^{4}$ National Center for Ecological Analysis and Synthesis, Univ. of Calif. Santa Barbara, Santa Barbara, CA 93101, USA. ${ }^{5}$ CyVerse, Thomas J. Keating Bioresearch Building, 1657 East Helen St, Tucson, AZ 85721, USA.

Received: 6 February 2016 Accepted: 3 September 2016

Published online: 23 September 2016

\section{References}

1. Buttigieg PL, Morrison N, Smith B, Mungall CJ, Lewis SE. The environment ontology: contextualising biological and biomedical entities. J Biomed Semant. 2013;4:43.

2. Field D, Amaral-Zettler L, Cochrane G, Cole JR, Dawyndt P, Garrity GM, Gilbert J, Glöckner FO, Hirschman L, Karsch-Mizrachi I, Klenk H-P, Knight R, Kottmann R, Kyrpides N, Meyer F, San Gil I, Sansone S-A, Schriml LM, Sterk P, Tatusova T, Ussery DW, White O, Wooley J. The Genomic Standards Consortium. PLoS Biol. 2011:9:e1001088.

3. Pesant S, Not F, Picheral M, Kandels-Lewis S, Le Bescot N, Gorsky G, ludicone D, Karsenti E, Speich S, Troublé R, Dimier C, Searson S. Open science resources for the discovery and analysis of Tara Oceans data. Sci Data. 2015;2(Lmd):150023.

4. Savio D, Sinclair L, ljaz UZ, Parajka J, Reischer GH, Stadler P, Blaschke AP, Blöschl G, Mach RL, Kirschner AKT, Farnleitner AH, Eiler A. Bacterial diversity along a $2600 \mathrm{~km}$ river continuum. Environ Microbiol. 2015;17(12):4994-5007.

5. Kopf A, Bicak M, Kottmann R, Schnetzer J, Kostadinov I, Lehmann K, Fernandez-Guerra A, Jeanthon C, Rahav E, Ullrich M, Wichels A, Gerdts G, Polymenakou P, Kotoulas G, Siam R, Abdallah RZ, Sonnenschein EC, Cariou T, O'Gara F, Jackson S, Orlic S, Steinke M, Busch J, Duarte B, Caçador I, CanningClode J, Bobrova O, Marteinsson V, Reynisson E, Loureiro CM, et al. The ocean sampling day consortium. Gigascience. 2015;4:27.

6. Leung MHY, Wilkins D, Li EKT, Kong FKF, Lee PKH. Indoor-air microbiome in an urban subway network: diversity and dynamics. Appl Env Microbiol. 2014:80:6760-70.

7. Hartmann M, Niklaus PA, Zimmermann S, Schmutz S, Kremer J, Abarenkov K, Lüscher P, Widmer F, Frey B. Resistance and resilience of the forest soil microbiome to logging-associated compaction. ISME J. 2014;8:226-44.

8. Meyer F, Paarmann D, D'souza M, Olson R, Glass EM, Kubal M, Paczian T, Rodriguez A, Stevens R, Wilke A. The metagenomics RAST server - a public resource for the automatic phylogenetic and functional analysis of metagenomes. BMC Bioinformatics. 2008;19:386. 
9. iMicrobe metagenomic record annotations with ENVO [https://github.com/ hurwitzlab/imicrobe-lib/blob/master/docs/mapping_files/CameraMetadata_ ENVO_working_copy.csv]. Accessed 14 Sept 2016.

10. Smith B, Ashburner M, Rosse C, Bard J, Bug W, Ceusters W, Goldberg LJ, Eilbeck K, Ireland A, Mungall CJ, Leontis N, Rocca-Serra P, Ruttenberg A, Sansone S-A, Scheuermann RH, Shah N, Whetzel PL, Lewis S. The OBO Foundry: coordinated evolution of ontologies to support biomedical data integration. Nat Biotechnol. 2007;25:1251-5.

11. Deck J, Barker K, Beaman R, Buttigieg PL, Dröge G, Miller C, Tuama ÉÓ Murrell Z, Parr C, Robbins B. Clarifying Concepts and Terms in Biodiversity Informatics. Stand Genomic Sci. 2013:8:352-9.

12. Walls RL, Guralnick R, Deck J, Buntzman A, Buttigieg PL, Davies N, Denslow MW, Gallery RE, Parnell JJ, Osumi-sutherland D, Robbins RJ. Meeting report : advancing practical applications of biodiversity ontologies. Stand Genomic Sci. 2014;9:1-10.

13. Thessen AE, Bunker DE, Buttigieg PL, Cooper LD, Dahdul WM, Domisch S, Franz NM, Jaiswal P, Lawrence-dill CJ, Midford PE, Mungall CJ, Ram J, Zhang G, Deans AR, Huala E, Lewis SE. Emerging semantics to link phenotype and environment. Peer J. 2015:3:e1470.

14. Walls RL, Deck J, Guralnick R, Baskauf S, Beaman R, Blum S, Bowers S, Buttigieg PL, Davies N, Endresen D, Gandolfo MA, Hanner R, Janning A, Krishtalka L, Matsunaga A, Midford P, Morrison N, Tuama ÉÓ, Schildhauer M, Smith B, Stucky BJ, Thomer A, Wieczorek J, Whitacre J, Wooley J. Semantics in Support of Biodiversity Knowledge Discovery: An Introduction to the Biological Collections Ontology and Related Ontologies. PLoS ONE. 2014;9:e89606.

15. Rocca-Serra P, Brandizi M, Maguire E, Sklyar N, Taylor C, Begley K, Field D, Harris S, Hide W, Hofmann O, Neumann S, Sterk P, Tong W, Sansone S-A. ISA software suite: supporting standards-compliant experimental annotation and enabling curation at the community level. Bioinformatics. 2010;26: 2354-6.

16. Wieczorek J, Bloom D, Guralnick R, Blum S, Döring M, Giovanni R, Robertson T, Vieglais D. Darwin Core: an evolving community-developed biodiversity data standard. PLOS ONE. 2012;7:e29715.

17. Wieczorek J, Bánki O, Blum S, Deck J, Döring M, Dröge G, Endresen D, Goldstein P, Leary P, Krishtalka L, Tuama ÉÓ, Robbins RJ, Robertson T, Yilmaz P. Meeting Report: GBIF hackathon-workshop on Darwin Core and sample data (22-24 May 2013). Stand Genomic Sci. 2014;9:585-98.

18. Henschel A, Anwar MZ, Manohar V. Comprehensive Meta-analysis of Ontology Annotated 16S rRNA Profiles Identifies Beta Diversity Clusters of Environmental Bacterial Communities. PLoS Comput Biol. 2015:11:e1004468.

19. Parr CS, Wilson N, Schulz K, Leary P, Hammock J, Rice J, Corrigan Jr. RJ. TraitBank: Practical semantics for organism attribute data. Semant Web Interoperability, Usability, Appl 2014, 650-1860.

20. Poelen JH, Simons JD, Mungall CJ. Global biotic interactions: An open infrastructure to share and analyze species-interaction datasets. Ecol Inf. 2014;24:148-59.

21. Xiang Z, Mungall CJ, Ruttenberg A, He Y. Ontobee: A Linked Data Server and Browser for Ontology Terms. In Proceedings of the 2nd International Conference on Biomedical Ontologies (ICBO). Volume 1. Buffalo: CEUR Workshop Proceedings (CEUR-WS.org); 2011. p. 279-281. http://ceur-ws.org/ Vol-833/.

22. Day-Richter J, Harris MA, Haendel M, Lewis S. OBO-Edit-an ontology editor for biologists. Bioinformatics. 2007;23:2198-200.

23. The Relations Ontology Code repository [https://github.com/oborel/oborelations]. Accessed 14 Sept 2016.

24. Basic Formal Ontology 2.0: Draft Specification and User's Guide [https:// github.com/BFO-ontology/BFO/raw/master/docs/bfo2-reference/BFO2Reference.pdf]. Accessed 14 Sept 2016.

25. The Environment Ontology Issue Tracker [https://github.com/ EnvironmentOntology/envo/issues]. Accessed 14 Sept 2016.

26. Overton JA, Dietze H, Essaid S, Osumi-sutherland D, Mungall CJ. ROBOT: A command-line tool for ontology development. In Proceedings of the International Conference on Biomedical Ontology (ICBO). Lisbon: CEUR Workshop Proceedings (CEUR-WS.org); 2015. p. 131-132. http://ceur-ws.org/ Vol-1515/

27. Courtot M, Gibson F, Lister AL, Malone J, Schober D, Brinkman RR, Ruttenberg A. MIREOT: the Minimum Information to Reference an External Ontology Term Institute of Medical Biometry and Medical Informatics (IMBI), University Medical. Appl Ontol. 2011;6:23-33.

28. Mungall CJ, Torniai C, Gkoutos GV, Lewis SE, Haendel MA. Uberon, an integrative multi-species anatomy ontology. Genome Biol. 2012;13:R5.
29. Degtyarenko K, de Matos P, Ennis M, Hastings J, Zbinden M, McNaught A, Alcántara R, Darsow M, Guedj M, Ashburner M. ChEBl: a database and ontology for chemical entities of biological interest. Nucleic Acids Res. 2008; 36(Database issue):D344-50.

30. Dietze H, Berardini TZ, Foulger RE, Hill DP, Lomax J, Osumi-Sutherland D, Roncaglia P, Mungall CJ. TermGenie - a web-application for pattern-based ontology class generation. J Biomed Semant. 2014;5:48.

31. Pafilis E, Frankild SP, Schnetzer J, Fanini L, Faulwetter S, Pavloudi C, Vasileiadou K, Leary P, Hammock J, Schulz K, Parr CS, Arvanitidis C, Jensen L. ENVIRONMENTS and EOL: identification of Environment Ontology terms in text and the annotation of the Encyclopedia of Life. Bioinformatics. 2015;31:1872-4.

32. Yilmaz $P$, Kottmann R, Field D, Knight $R$, Cole JR, Amaral-Zettler L, Gilbert JA, Karsch-Mizrachi I, Johnston A, Cochrane G, Vaughan R, Hunter C, Park J, Morrison N, Rocca-Serra P, Sterk P, Arumugam M, Bailey M, Baumgartner L, Birren BW, Blaser MJ, Bonazzi V, Booth T, Bork P, Bushman FD, Buttigieg PL Chain PSG, Charlson E, Costello EK, Huot-Creasy H, et al. Minimum information about a marker gene sequence (MIMARKS) and minimum information about any $(X)$ sequence (MIXS) specifications. Nat Biotechnol. 2011;29:415-20.

33. Glass EM, Dribinsky $Y$, Yilmaz $P$, Levin $H$, Van Pelt $R$, Wendel $D$, Wilke $A$, Eisen JA, Huse S, Shipanova A, Sogin M, Stajich J, Knight R, Meyer F, Schriml LM. MIXS-BE: a MIXS extension defining a minimum information standard for sequence data from the built environment. ISME J. 2014;8:1-3.

34. ten Hoopen $\mathrm{P}$, Amid C, Buttigieg PL, Pafilis E, Bravakos $\mathrm{P}$, Cerdeño-Tárraga AM, Gibson R, Kahlke T, Legaki A, Murthy KN, Papastefanou G, Pereira E, Rossello M, Toribio AL, Cochrane G. Value, but high costs in post-deposition data curation. Database 2016:1-10. doi: 10.1093/database/bav126.

35. Lund HG. What is a forest? Definitions do make a difference: An example from Turkey. Avrasya Terim Derg. 2014;2:1-8.

36. Sasaki N, Putz FE. Critical need for new definitions of "forest" and "forest degradation" in global climate change agreements. Conserv Lett. 2009;2:226-32.

37. Sexton JO, Noojipady P, Song X-P, Feng M, Song D-X, Kim D-H, Anand A, Huang C, Channan S, Pimm SL, Townshend JR. Conservation policy and the measurement of forests. Nat Clim Chang. 2015;6(October):1-6.

38. Mungall CJ, Bada M, Berardini TZ, Deegan J, Ireland A, Harris MA, Hill DP, Lomax J. Cross-product extensions of the Gene Ontology. J Biomed Semant Inf. 2011;44:80-6.

39. Parr CS, Wilson N, Leary P, Schulz KS, Lans K, Walley L, Hammock JA, Goddard A, Rice J, Studer M, Holmes JTG, Corrigan RJ. The encyclopedia of life v2: providing global access to knowledge about life on earth. Biodiv Data J. 2014:29(2):e1079.

40. The Tree of Life Web Project [http://tolweb.org]. Accessed 14 Sept 2016.

41. Huerta-Cepas J, Capella-Gutierrez S, Pryszcz LP, Marcet-Houben M, Gabaldon T. PhylomeDB v4: zooming into the plurality of evolutionary histories of a genome. Nucleic Acids Res. 2014;42:D897-902.

42. Piel WH, Donoghue MJ, Sanderson MJ. TreeBASE: a database of phylogenetic knowledge. In: Shimura J, Wilson KL, Gordon D, editors. To the interoperable "Catalog of Life" with partners Species 2000 Asia Oceanea. Research report no. 171. Tsukuba, Japan: The National Institute for Environmental Studies; 2002. p. 41-7.

43. Vos R, Lapp H, Piel W, Tannen V. TreeBASE2: Rise of the Machines. Available from Nature Precedings; 2010.

44. IUCN: The IUCN Red List of Threatened Species. Version 2015-4 2015:http:// www.iucnredlist.org. Accessed 14 Sept 2016

45. The Environment Ontology's experimental habitat branch [https://github. com/EnvironmentOntology/envo-habitats]. Accessed 14 Sept 2016.

46. Experimental ENVO Habitat Classes Derived from ENVIRONMENTS-EOL [http://dx.doi.org/10.5281/zenodo.35393]. Accessed 14 Sept 2016.

47. Groom QJ. Piecing together the biogeographic history of Chenopodium vulvaria L. using botanical literature and collections. Peer J. 2015:3:e723.

48. Elith J, Leathwick JR. Species distribution models: ecological explanation and prediction across space and time. Ann Rev Ecol Evol Syst. 2009;40:677-97.

49. Hall LS, Krausman PR, Morrison ML. The habitat concept and a plea for standard terminology. Wildl Soc Bull. 1997;25:173-82.

50. Kearney M. Habitat, environment and niche: What are we modelling? Oikos 2006;115:186-91.

51. Schmidt TSB, Matias Rodrigues JF, von Mering C. Limits to robustness and reproducibility in thedemarcation of operational taxonomic units. Env Microbiol. 2014:17:1689-706

52. Schmidt TSB, Matias Rodrigues JF, von Mering C. Ecological consistency of SSU rRNA-based operational taxonomic units at a global scale. PLoS Comput Biol. 2014;10:e1003594 
53. Mahé F, Rognes T, Quince C, de Vargas C, Dunthorn M. Swarm: robust and fast clustering method for amplicon-based studies. Peer J. 2014;2:e593.

54. Eren AM, Maignien L, Sul WJ, Murphy LG, Grim SL, Morrison HG, Sogin ML. Oligotyping: differentiating between closely related microbial taxa using $16 \mathrm{~S}$ rRNA gene data. Methods Ecol Evol. 2013:4:1111-9.

55. Eren a M, Morrison HG, Lescault PJ, Reveillaud J, Vineis JH, Sogin ML. Minimum entropy decomposition: Unsupervised oligotyping for sensitive partitioning of high-throughput marker gene sequences. ISME J. 2014;9:968-79.

56. Ellis EC, Ramankutty N. Putting people in the map: anthropogenic biomes of the world. Front Ecol Env. 2008;6:439-47.

57. Talisuna A, Adibaku S, Dorsey G, Kamya MR, Rosenthal PJ. Malaria in Uganda: challenges to control on the long road to elimination. II. The path forward. Acta Trop. 2012;121:196-201.

58. Wanzirah H, Tusting LS, Arinaitwe E, Katureebe A, Maxwell K, Rek J, Bottomley C, Staedke SG, Kamya M, Dorsey G, Lindsay SW. Mind the gap: house structure and the risk of malaria in Uganda. PLOS ONE. 2015;10: e0117396.

59. Pletscher-Frankild S, Pallejà A, Tsafou K, Binder JX, Jensen ப. DISEASES: Text mining and data integration of disease-gene associations. Methods. 2014;74:83-9.

60. Groza T, Köhler S, Doelken S, Collier N, Oellrich A, Smedley D, Couto FM, Baynam G, Zankl A, Robinson PN. Automatic concept recognition using the human phenotype ontology reference and test suite corpora. Database (Oxford). 2015:2015:1-13

61. Tringe SG, Zhang T, Liu X, Yu Y, Lee WH, Yap J, Yao F, Suan ST, Ing SK, Haynes M, Rohwer F, Wei CL, Tan P, Bristow J, Rubin EM, Ruan Y. The Airborne Metagenome in an Indoor Urban Environment. PLoS ONE. 2008;3:10.

62. Hospodsky D, Qian J, Nazaroff WW, Yamamoto N, Bibby K, Rismani-Yazdi H, Peccia J. Human occupancy as a source of indoor airborne bacteria. PLoS ONE. 2012;7:e34867.

63. Adams Rl, Bateman AC, Bik HM, Meadow JF. Microbiota of the indoor environment: a meta-analysis. Microbiome. 2015;3:49.

64. Flores GE, Bates ST, Knights D, Lauber CL, Stombaugh J, Knight R, Fierer N. Microbial biogeography of public restroom surfaces. PLOS ONE. 2011;6:e28132.

65. Dunn RR, Fierer N, Henley JB, Leff JW, Menninger HL. Home life: factors structuring the bacterial diversity found within and between homes. PLoS ONE. 2013;8:e64133.

66. Hastings J, Jeliazkova N, Owen G, Tsiliki G, Munteanu CR, Steinbeck C, Willighagen E. eNanoMapper: harnessing ontologies to enable data integration for nanomaterial risk assessment. J Biomed Semantics. 2015;6:10.

67. Lovett GM, Tear TH, Evers DC, Findlay SEG, Cosby BJ, Dunscomb JK, Driscoll CT, Weathers KC. Effects of air pollution on ecosystems and biological diversity in the eastern United States. Ann N Y Acad Sci. 2009:1162:99-135.

68. The Sustainable Development Goals Interface Ontology (SDGIO) Code Repository [https://github.com/SDG-InterfaceOntology/sdgio]. Accessed 14 Sept 2016

69. Colglazier W. SUSTAINABILITY. Sustainable development agenda: 2030 Sci (80-). 2015;349:1048-50

70. UNEP. Embedding the Environment in Sustainable Development Goals. UNEP Post-2015 Discussion Paper 1. Nairobi: United Nations Environment Programme (UNEP); 2013.

71. United Nations. Transforming our world: the 2030 agenda for sustainable development. 2015:A/RES/70/1

72. General Multilingual Environmental Thesaurus (GEMET). 2012:version 3.1. http://www.eionet.europa.eu/gemet/. Accessed 14 Sept 2016.

73. Schmitt CB, Belokurov A, Besançon C, Boisrobert L, Burgess ND, Campbell A, Coad L, Fish L, Gliddon D, Humphries K, Kapos V, Loucks C, Lysenko I, Miles L, Mills C, Minnemeyer S, Pistorius T, Ravilious C, Steininger M, Winkel G. Global Ecological Forest Classification and Forest Protected Area Gap Analysis. Analyses and Recommendations in View of the 10\% Target for Forest Protection under the Convention on Biological Diversity (CBD). 2nd ed. Freiburg: University Press; 2009.

74. Soltwedel T, Schauer U, Boebel O, Nothig EM, Bracher A, Metfies K, Schewe I, Boetius A, Klages M. FRAM - FRontiers in Arctic marine Monitoring Visions for permanent observations in a gateway to the Arctic Ocean. In OCEANS 2013 MTS/IEEE: The Challenges of the Northern Dimension. Bergen: IEEE; 2013. http://ieeexplore.ieee.org/document/6608008/?arnumber $=6608008$

75. The Principles of the OBO Foundry [http://www.obofoundry.org/principles/ fp-000-summary.html]. Accessed 14 Sept 2016

76. The Food Ontology (FOODON) Code Repository [https://github.com/ FoodOntology/foodon]. Accessed 14 Sept 2016
77. The Agrononomy Ontology (AgrO) code repository [https://github.com/ AgriculturalSemantics/agro].

78. CEM-IUCN, Provita. IUCN Red List of Ecosystems. Caracas, Venez: Com Ecosyst Manag Int Union Conserv Nat Provita; 2012. http://www. iucnredlistofecosystems.org.

79. Hardisty A, Roberts D, Biodiversity Informatics Community. A decadal view of biodiversity informatics: challenges and priorities. BMC Ecol. 2013;13:16.

80. Wilkinson MD, Dumontier M, Aalbersberg IJ, Appleton G, Axton M, Baak A Blomberg N, Boiten J-W, da Silva Santos LB, Bourne PE, Bouwman J, Brookes AJ, Clark T, Crosas M, Dillo I, Dumon O, Edmunds S, Evelo CT, Finkers R, Gonzalez-Beltran A, Gray AJG, Groth P, Goble C, Grethe JS, Heringa J, 't Hoen PA, Hooft R, Kuhn T, Kok R, Kok J, et al. The FAIR guiding principles for scientific data management and stewardship. Sci Data. 2016;3:160018.

81. Shaping the semantic layer by mining digitised data: an encounter between iDigBio's plant records and the Environment Ontology (ENVO) [https://www.idigbio.org/content/webinar-shaping-semantic-layer-miningdigitised-data-encounter-between-idigbios-plant]. Accessed 14 Sept 2016.

82. The Environment Ontology (OWL representation) [http://purl.obolibrary.org/ obo/envo.owl]. Accessed 14 Sept 2016.

\section{Submit your next manuscript to BioMed Central and we will help you at every step:}

- We accept pre-submission inquiries

- Our selector tool helps you to find the most relevant journal

- We provide round the clock customer support

- Convenient online submission

- Thorough peer review

- Inclusion in PubMed and all major indexing services

- Maximum visibility for your research

Submit your manuscript at www.biomedcentral.com/submit

) Biomed Central 